\title{
MAXIMUM NORM A POSTERIORI ERROR ESTIMATE FOR A 2D SINGULARLY PERTURBED SEMILINEAR REACTION-DIFFUSION PROBLEM*
}

\author{
NATALIA KOPTEVA ${ }^{\dagger}$
}

\begin{abstract}
A singularly perturbed semilinear reaction-diffusion equation, posed in the unit square, is discretized on arbitrary nonuniform tensor-product meshes. We establish a second-order maximum norm a posteriori error estimate that holds true uniformly in the small diffusion parameter. No mesh aspect ratio assumption is made. Numerical results are presented that support our theoretical estimate.
\end{abstract}

Key words. reaction-diffusion, singular perturbation, a posteriori error estimate, maximum norm, no mesh aspect ratio condition, finite differences, layer-adapted mesh

AMS subject classifications. $65 \mathrm{~N} 06,65 \mathrm{~N} 15,65 \mathrm{~N} 50$

DOI. $10.1137 / 060677616$

1. Introduction. Solutions of singularly perturbed differential equations typically exhibit sharp boundary and interior layers, which are narrow regions where solutions change rapidly. To obtain reliable numerical approximations of layer solutions in an efficient way, one has to use locally refined meshes that are fine in layer regions and standard outside. Furthermore, as is shown in $[20,19,7,13]$ by the numerical analysis of model problems, for which the location and width of the layers are known a priori, optimal layer-adapted meshes have extremely high maximum aspect ratios (typically $O\left(\varepsilon^{-1}\right)$, where $\varepsilon$ is the layer width).

In contrast, a posteriori error estimates, which underlie any reliable a posteriori mesh construction, are typically obtained under the minimum angle condition, which is equivalent to the bounded-mesh-aspect-ratio condition; see, e.g., [2, 21]. But the minimum angle condition seems rather restrictive and makes a posteriori error estimates less practical for layer solutions, for which a posteriori mesh generation is most needed.

The aim of the present paper is to establish an a posteriori error estimate for one singularly perturbed problem under no mesh aspect ratio condition. Note that our error estimate is in the maximum norm, which is sufficiently strong to capture layers and hence seems most appropriate for singularly perturbed problems.

Consider the singularly perturbed semilinear reaction-diffusion problem posed in the unit square:

$$
\begin{aligned}
T u:=-\varepsilon^{2} \triangle u+b(x, y, u) & =0, & & (x, y) \in \Omega=(0,1) \times(0,1), \\
u(x, y) & =0, & & (x, y) \in \partial \Omega .
\end{aligned}
$$

\footnotetext{
* Received by the editors December 14, 2006; accepted for publication (in revised form) August 21, 2007; published electronically April 11, 2008. This publication emanated from research conducted with the financial support of Science Foundation Ireland under the Basic Research Grant Programme 2004, grant 04/BR/M0055.

http://www.siam.org/journals/sinum/46-3/67761.html

$\dagger$ Department of Mathematics and Statistics, University of Limerick, Limerick, Ireland (natalia. kopteva@ul.ie).
} 
Here $\varepsilon$ is a small positive parameter, $\triangle=\partial^{2} / \partial x^{2}+\partial^{2} / \partial y^{2}$ is the Laplace operator, the function $b$ is sufficiently smooth, and

$$
0<\beta<b_{u}(x, y, u) \leq \bar{\beta} \quad \text { for all }(x, y, u) \in[0,1]^{2} \times \mathbb{R} .
$$

Under condition (1.2), problem (1.1) has a unique solution, which exhibits sharp boundary layers of width $O(\varepsilon|\ln \varepsilon|)$ along the boundary $\partial \Omega$.

We discretize (1.1) using the standard second-order five-point difference schemesee (2.2) for details - on an arbitrary tensor-product mesh $\left\{\left(x_{i}, y_{j}\right)\right\}$, where $0=x_{0}<$ $x_{1}<\cdots<x_{N}=1$ and $0=y_{0}<y_{1}<\cdots<y_{M}=1$, while $h_{i}=x_{i}-x_{i-1}$ and $k_{j}=y_{j}-y_{j-1}$ are the local mesh sizes.

This is an idealized situation in the a posteriori mesh construction context since an irregular mesh, rather than a tensor-product mesh, seems more suitable for a practical a posteriori mesh construction algorithm. Therefore the error estimate, which we present, is more interesting from a theoretical point of view. In particular, it shows that the bounded-mesh-aspect-ratio condition/minimal-angle condition is not essential in the a posteriori error estimation. Furthermore, if tensor-product meshes are used at least in crucial layer regions, where the mesh adaptation is most needed, one might conjecture that in such regions, local analogues of our a posteriori error estimate would apply.

Our main result is the following maximum norm a posteriori error estimate, in which the error is understood as the difference between the exact solution and the bilinear interpolant of the computed solution:

$$
\left\|U^{\mathrm{B}}-u\right\|_{\infty} \leq C_{0}\left[\max _{\substack{i=1, \ldots, N \\ j=0, \ldots, M}}\left\{h_{i}^{2} M_{1, i j}\right\}+\max _{\substack{i=0, \ldots, N \\ j=1, \ldots, M}}\left\{k_{j}^{2} M_{2, i j}\right\}\right]
$$

—see Theorem 2.1 - where, roughly speaking,

$$
M_{1, i j} \approx\left|D_{x}^{2} U_{i j}\right| \ln (2+\varepsilon / \kappa)+1, \quad M_{2, i j} \approx\left|D_{y}^{2} U_{i j}\right| \ln (2+\varepsilon / \kappa)+1,
$$

with $\kappa:=\min \left\{\min _{i}\left\{h_{i}\right\}, \min _{j}\left\{k_{j}\right\}\right\}$. Here $U^{\mathrm{B}}$ is the bilinear interpolant of the computed solution $U$ (the finite difference computed solution is originally defined at the mesh nodes only; hence to measure the error in the entire domain, one first has to interpolate the computed solution there). The quantities $D_{x}^{2} U_{i j}$ and $D_{y}^{2} U_{i j}$ are the standard discrete approximations of $\partial^{2} u / \partial x^{2}$ and $\partial^{2} u / \partial y^{2}$ defined in (2.3). In (1.4), a few terms are skipped, for which the one-dimensional analysis [12] and the numerical results of section 6 show that they are less important; see Theorem 2.1 for the precise definitions of $M_{1, i j}$ and $M_{2, i j}$.

The error constant $C_{0}$ in (1.3) is independent of $\varepsilon$, the mesh, and aspect ratios of its elements, although this constant is not specified. In a posteriori error estimation, much attention focuses on specifying the error constants. Note that for singularly perturbed problems, the error constant might blow up as $\varepsilon$ becomes small, and hence the existence of an $\varepsilon$-uniform error constant is more significant than its precise value.

Note that, roughly speaking, the a posteriori error estimate $(1.3),(1.4)$ might be viewed as a discrete analogue of the linear interpolation error estimates $[8,6]$, which imply that a suitable anisotropic mesh should be quasi-uniform under the metric induced by the Hessian matrix. An example of this idea being exploited for mesh generation is given, e.g., in [10], where anisotropic meshes, defined as mappings of regular uniform grids, are obtained via functional minimization. 
The present paper follows [11] and in particular [12], where certain maximum norm a posteriori error estimates were derived for one-dimensional singularly perturbed convection-diffusion and reaction-diffusion problems; see also a more recent paper [18] for a similar one-dimensional a posteriori error estimate. Note that the papers $[11,12,18]$ report one-dimensional error estimates, while now we extend the one-dimensional analysis [12] to a two-dimensional case.

Problem (1.1) has often been addressed in the numerical analysis literature. In particular, we refer the reader to [5], where iterative techniques for the standard finite difference discretization of (1.1) are developed, and [15, 16], where certain a posteriori error estimates for a linear version of (1.1) are obtained on anisotropic meshes in the energy norm.

The paper is organized as follows. In section 2, we describe the numerical method, present our a posteriori error estimate in Theorem 2.1, and outline its proof. Next, in section 3, we establish some estimates for the Green's function of a linearized version of (1.1). They imply certain stability properties of the differential operator $T$ from (1.1), which are presented in section 4. Then in section 5, we complete the proof of Theorem 2.1. Finally, in section 6, numerical results are given that support our theoretical estimate.

Notation. Let $\|\cdot\|_{p ; \tilde{\Omega}}$, where $1 \leq p \leq \infty$, denote the standard $L_{p}(\tilde{\Omega})$ norm for any domain $\tilde{\Omega}$. Furthermore, the standard notation $W^{k, p}(\tilde{\Omega})$ is used for the Sobolev spaces with the norm $\|\cdot\|_{k, p ; \tilde{\Omega}}$ defined, for a function $v(x, y)$ in a domain $\tilde{\Omega}$, by

$$
\begin{gathered}
\|v\|_{k, p ; \tilde{\Omega}}=\|v\|_{p ; \tilde{\Omega}}+\sum_{l=1}^{k}|v|_{l, p ; \tilde{\Omega}}, \quad k=1,2, \\
|v|_{1, p ; \tilde{\Omega}}=\left\|v_{x}\right\|_{p ; \tilde{\Omega}}+\left\|v_{y}\right\|_{p ; \tilde{\Omega}}, \quad|v|_{2, p ; \tilde{\Omega}}=\left\|v_{x x}\right\|_{p ; \tilde{\Omega}}+\left\|v_{x y}\right\|_{p ; \tilde{\Omega}}+\left\|v_{y y}\right\|_{p ; \tilde{\Omega}} ;
\end{gathered}
$$

see, e.g., [9]. We shall use the notation $\|\cdot\|_{p}$ and $\|\cdot\|_{k, p}$ for $\|\cdot\|_{p ; \Omega}$ and $\|\cdot\|_{k, p ; \Omega}$ when there is no ambiguity. Sometimes the domain of interest will be an open ball $B(a, b ; \rho)=\left\{(x, y):(x-a)^{2}+(y-b)^{2}<\rho^{2}\right\}$ centered at $(a, b)$ of radius $\rho$.

Throughout the paper we let $C$ denote a generic positive constant that may take different values in different formulas but is always independent of the mesh and $\varepsilon$. A subscripted $C$ (e.g., $C_{1}$ ) denotes a positive constant that is independent of $h$ and $\varepsilon$ and takes a fixed value.

Remark 1.1. The assumption $b_{u}(x, y, u) \leq \bar{\beta}$ in (1.2) can be omitted since it follows, for some constant $\bar{\beta}$, from $0<\beta<b_{u}(x, y, u)$ and $u$ being a unique and bounded solution of (1.1); see, e.g., [22, section 12]. Note that assumption (1.2) enables us to linearize (1.1) and then invoke the Green's function in our analysis. On the other hand, this assumption implies that our problem has a unique solution and thus excludes the possibility of multiple solutions. Hence, strictly speaking, our results are not applicable to the multiple-solution case. Having said this, we still believe that the present paper provides some insight into the numerical solution of a more general multiple-solution version of (1.1), considered, e.g., in [10].

2. Numerical method. Main result. Let our problem (1.1) satisfy the standard compatibility conditions at the corners of the domain $\Omega$ :

$$
b(0,0,0)=b(0,1,0)=b(1,0,0)=b(1,1,0)=0,
$$

which guarantee that $u \in C^{3}(\bar{\Omega})$. 
Numerical method. We require the computed solution $U$ to satisfy the standard five-point finite difference discretization of problem (1.1):

$$
-\varepsilon^{2} D_{x}^{2} U_{i j}-\varepsilon^{2} D_{y}^{2} U_{i j}+b\left(x_{i}, y_{j}, U_{i j}\right)=0
$$

for $i=1, \ldots N-1, j=1, \ldots, M-1$, where $U_{0, j}=U_{N, j}=U_{i, 0}=U_{i, M}=0$. Here, as usual, $U_{i j}$ is associated with the mesh node $\left(x_{i}, y_{j}\right)$, and we use the standard finite difference operators, defined for a discrete function $V_{i j}$ by

$$
\begin{array}{ll}
D_{x}^{-} V_{i j}=\frac{V_{i j}-V_{i-1, j}}{h_{i}}, & D_{x}^{2} V_{i j}=\frac{D_{x}^{-} V_{i+1, j}-D_{x}^{-} V_{i, j}}{\left(h_{i}+h_{i+1}\right) / 2}, \\
D_{y}^{-} V_{i j}=\frac{V_{i j}-V_{i, j-1}}{k_{j}}, & D_{y}^{2} V_{i j}=\frac{D_{y}^{-} V_{i, j+1}-D_{y}^{-} V_{i, j}}{\left(k_{j}+k_{j+1}\right) / 2} .
\end{array}
$$

By condition (1.2), there exists a unique solution of the discrete problem (2.2) on an arbitrary mesh $\left\{\left(x_{i}, y_{j}\right)\right\}$; see, e.g., [5].

Clearly, $D_{x}^{2} U_{i j}$ is defined for $i=1, \ldots, N-1, j=0, \ldots, M$, while $D_{y}^{2} U_{i j}$ is defined for $i=0, \ldots, N, j=1, \ldots, M-1$. We now extend $D_{x}^{2} U_{i j}$ to the mesh nodes $i=0, N$ as follows. First, formally extend the discrete equation (2.2) to $i=0$ and $i=N$, in which, using the zero boundary conditions, we set $D_{y}^{2} U_{0, j}=D_{y}^{2} U_{N, j}=0$. This yields

$$
D_{x}^{2} U_{0, j}:=\varepsilon^{-2} b\left(0, y_{j}, 0\right), \quad D_{x}^{2} U_{N, j}:=\varepsilon^{-2} b\left(1, y_{j}, 0\right), \quad j=0, \ldots, M .
$$

Similarly, extend $D_{x}^{2} U_{i j}$ to $j=0, M$ by

$$
D_{y}^{2} U_{i, 0}:=\varepsilon^{-2} b\left(x_{i}, 0,0\right), \quad D_{y}^{2} U_{i, M}:=\varepsilon^{-2} b\left(x_{i}, 1,0\right), \quad i=0, \ldots, N .
$$

Note that by (2.1), the above relations (2.4) imply that $D_{x}^{2} U_{i j}=D_{y}^{2} U_{i j}=0$ if $\left(x_{i}, y_{j}\right)$ is $(0,0),(0,1),(1,0)$, or $(1,1)$, which is consistent with the boundary condition in (1.1).

Remark 2.1. Now that $D_{x}^{2} U_{i j}$ and $D_{y}^{2} U_{i j}$ are extended by (2.4) to all $i, j$, our discrete equation (2.2) holds true for all $i=0, \ldots, N$ and $j=0, \ldots, M$.

Bilinear interpolation notation. Let $U^{\mathrm{B}}=U^{\mathrm{B}}(x, y)$ be the standard bilinear interpolant of the computed solution $U_{i j}$; i.e., $U^{\mathrm{B}}$ is continuous in $\bar{\Omega}$, bilinear on each $\left[x_{i-1}, x_{i}\right] \times\left[y_{j-1}, y_{j}\right]$, and equal to $U_{i j}$ at the mesh nodes:

$$
U^{\mathrm{B}}\left(x_{i}, y_{j}\right)=U_{i j} \quad \text { for } \quad i=0, \ldots, N, j=0, \ldots, M .
$$

Similarly, we introduce the bilinear interpolant $v^{\mathrm{B}}(x, y)$ for any discrete function $v_{i j}$ or any continuous function $v(x, y)$.

Furthermore, we shall use the standard one-dimensional linear interpolants $v^{\mathrm{I}}$ and $v^{\mathrm{J}}$ with respect to $x$ and $y$, respectively, that are defined, for any function $v$, as follows. For each fixed $y$ in the domain of $v$, we have $v^{\mathrm{I}}\left(x_{i}, y\right)=v\left(x_{i}, y\right)$, and $v^{\mathrm{I}}(x, y)$ is linear on each $\left[x_{i-1}, x_{i}\right]$. Similarly, for each fixed $x$ in the domain of $v$, we have $v^{\mathrm{J}}\left(x, y_{j}\right)=v\left(x, y_{j}\right)$, and $v^{\mathrm{J}}(x, y)$ is linear on each $\left[y_{j-1}, y_{j}\right]$.

In particular, consider the bilinear interpolant $U^{\mathrm{B}}$ of $U_{i j}$ and the linear onedimensional interpolants $U^{\mathrm{I}}\left(x, y_{j}\right)$ and $U^{\mathrm{J}}\left(x_{i}, y\right)$. Clearly,

$$
\left[U^{\mathrm{I}}\left(x, y_{j}\right)\right]^{\mathrm{J}}=\left[U^{\mathrm{J}}\left(x_{i}, y\right)\right]^{\mathrm{I}}=U^{\mathrm{B}}(x, y) .
$$

Now we state a maximum norm a posteriori error estimate, which is the main result of the present paper. 
TheOrem 2.1. Let $u(x, y)$ be a solution of problem (1.1), (1.2), (2.1), $U_{i j}$ a solution of problem $(2.2)$ on an arbitrary mesh $\left\{\left(x_{i}, y_{j}\right)\right\}$, and $U^{\mathrm{B}}(x, y)$ its piecewise bilinear interpolant (2.5). Then

$$
\left\|U^{\mathrm{B}}-u\right\|_{\infty} \leq C_{0}\left[\max _{\substack{i=1, \ldots, N \\ j=0, \ldots, M}}\left\{h_{i}^{2} M_{1, i j}\right\}+\max _{\substack{i=0, \ldots, N \\ j=1, \ldots, M}}\left\{k_{j}^{2} M_{2, i j}\right\}\right],
$$

where

$$
\begin{aligned}
& M_{1, i j}:=\min \left\{\left|D_{x}^{2} U_{i-1, j}\right|,\left|D_{x}^{2} U_{i j}\right|\right\} \ln (2+\varepsilon / \kappa)+\varepsilon\left|D_{x}^{-} D_{x}^{2} U_{i j}\right|+\left|D_{x}^{-} U_{i j}\right|^{2}+1, \\
& M_{2, i j}:=\min \left\{\left|D_{y}^{2} U_{i, j-1}\right|,\left|D_{y}^{2} U_{i j}\right|\right\} \ln (2+\varepsilon / \kappa)+\varepsilon\left|D_{y}^{-} D_{y}^{2} U_{i j}\right|+\left|D_{y}^{-} U_{i j}\right|^{2}+1,
\end{aligned}
$$

with $\kappa:=\min \left\{\min _{i}\left\{h_{i}\right\}, \min _{j}\left\{k_{j}\right\}\right\}$, while the constant $C$ is independent of $\varepsilon$ and the mesh.

Proof outline. By (1.1), we have

$$
T U^{\mathrm{B}}-T u=-\varepsilon^{2}\left[\partial^{2} / \partial x^{2}+\partial^{2} / \partial y^{2}\right] U^{\mathrm{B}}+b\left(x, y, U^{\mathrm{B}}\right),
$$

where $\partial^{2} U^{\mathrm{B}} / \partial x^{2}$ and $\partial^{2} U^{\mathrm{B}} / \partial y^{2}$ are understood in the sense of distributions. Define an auxiliary function

$$
q(x, y):=b\left(x, y, U^{\mathrm{B}}(x, y)\right)
$$

and let $q^{\mathrm{B}}$ denote its piecewise bilinear interpolant on the mesh $\left\{\left(x_{i}, y_{j}\right)\right\}$. Hence

$$
T U^{\mathrm{B}}-T u=-\varepsilon^{2}\left[\partial^{2} / \partial x^{2}+\partial^{2} / \partial y^{2}\right] U^{\mathrm{B}}+q^{\mathrm{B}}+\left[q-q^{\mathrm{B}}\right] .
$$

Noting that $q_{i j}:=q\left(x_{i}, y_{j}\right)=b\left(x_{i}, y_{j}, U_{i j}\right)$ and recalling the discrete equation (2.2) combined with Remark 2.1 yields $q_{i j}=\varepsilon^{2} D_{x}^{2} U_{i j}+\varepsilon^{2} D_{y}^{2} U_{i j}$ for $i=0, \ldots, N, j=$ $0, \ldots, M$. Next, decompose this as $q_{i j}=q_{1, i j}+q_{2, i j}$, where

$$
q_{1, i j}:=\varepsilon^{2} D_{x}^{2} U_{i j}, \quad q_{2, i j}:=\varepsilon^{2} D_{y}^{2} U_{i j}, \quad i=0, \ldots, N, j=0, \ldots, M .
$$

Furthermore, using analogues of (2.6) for $q_{1}$ and $q_{2}$, we get

$$
q^{\mathrm{B}}(x, y)=q_{1}^{\mathrm{B}}(x, y)+q_{2}^{\mathrm{B}}(x, y)=\left[q_{1}^{\mathrm{I}}\left(x, y_{j}\right)\right]^{\mathrm{J}}+\left[q_{2}^{\mathrm{J}}\left(x_{i}, y\right)\right]^{\mathrm{I}}, \quad(x, y) \in \bar{\Omega} .
$$

Therefore

$T U^{\mathrm{B}}-T u=\left[-\varepsilon^{2} \frac{\partial^{2} U^{\mathrm{I}}\left(x, y_{j}\right)}{\partial x^{2}}+q_{1}^{\mathrm{I}}\left(x, y_{j}\right)\right]^{\mathrm{J}}+\left[-\varepsilon^{2} \frac{\partial^{2} U^{\mathrm{J}}\left(x_{i}, y\right)}{\partial y^{2}}+q_{2}^{\mathrm{J}}\left(x_{i}, y\right)\right]^{\mathrm{I}}+\left[q-q^{\mathrm{B}}\right]$.

Here we used

$$
\frac{\partial^{2} U^{\mathrm{B}}(x, y)}{\partial x^{2}}=\left[\frac{\partial^{2} U^{\mathrm{I}}\left(x, y_{j}\right)}{\partial x^{2}}\right]^{\mathrm{J}}, \quad \frac{\partial^{2} U^{\mathrm{B}}(x, y)}{\partial y^{2}}=\left[\frac{\partial^{2} U^{\mathrm{J}}\left(x_{i}, y\right)}{\partial y^{2}}\right]^{\mathrm{I}},
$$

which follow from (2.6); see also Remark 2.2.

The proof is completed in section 5 . First, the residual $T U^{\mathrm{B}}-T u$ is represented as

$$
T U^{\mathrm{B}}-T u=\frac{\partial}{\partial x} F_{1}(x, y)+\frac{\partial}{\partial y} F_{2}(x, y)+\left[q-q^{\mathrm{B}}\right],
$$

Copyright (c) by SIAM. Unauthorized reproduction of this article is prohibited. 
where $F_{1}$ and $F_{2}$ are functions of the current mesh and computed solution. This will enable us to estimate the error $U^{\mathrm{B}}-u$ in the maximum norm by linearizing the operator $T$ and invoking its stability properties, which are obtained in section 4 using sharp estimates of the Green's function of section 3.

Remark 2.2. We understand $\partial^{2} U^{\mathrm{B}} / \partial x^{2}$ and $\partial^{2} U^{\mathrm{B}} / \partial y^{2}$ in the sense of distributions. To be more precise, in (2.9) we use $\partial^{2} U^{\mathrm{I}}\left(x, y_{j}\right) / \partial x^{2}=\sum_{i=1}^{N-1} v_{i}\left(y_{j}\right) \delta\left(x-x_{i}\right)$, and $\left[\partial^{2} U^{\mathrm{I}}\left(x, y_{j}\right) / \partial x^{2}\right]^{\mathrm{J}}=\sum_{i=1}^{N-1} v_{i}^{\mathrm{J}}(y) \delta\left(x-x_{i}\right)$, where $\delta(\cdot)$ is the Dirac $\delta$-distribution, while $v_{i}\left(y_{j}\right):=D^{2} U_{i j}\left(h_{i}+h_{i+1}\right) / 2$.

3. Green's function. Assumption (1.2) enables us to linearize (1.1) and then invoke the Green's function in our analysis. Hence we start with a linear case of (1.1), where $b(x, y, u):=p(x, y) u-f(x, y)$ :

$$
L u:=-\varepsilon^{2} \triangle u+p(x, y) u=f(x, y) \text { in } \Omega, \quad u=0 \quad \text { on } \partial \Omega .
$$

Here $p \in L_{\infty}(\Omega)$ and, in accordance with (1.2),

$$
0<\beta \leq p(x, y) \leq \bar{\beta} .
$$

Introduce the Green's function $G(x, y ; \xi, \eta)$ of the linear self-adjoint operator $L$ that, for each $(x, y) \in \Omega$, satisfies

$$
\begin{aligned}
L G=-\varepsilon^{2}\left[G_{\xi \xi}+G_{\eta \eta}\right]+p(\xi, \eta) G & =\delta(x-\xi) \delta(y-\eta), \quad(\xi, \eta) \in \Omega, \\
G(x, y ; \xi, \eta) & =0, \quad(\xi, \eta) \in \partial \Omega,
\end{aligned}
$$

where $\delta(\cdot)$ is the Dirac $\delta$-distribution. Then the unique solution $u$ of problem (3.1) is

$$
u(x, y)=\int_{\Omega} G(x, y ; \xi, \eta) f(\xi, \eta) d \xi d \eta
$$

Starting from (3.3), throughout the present section, the differential operators $L$ and $\bar{L}$ are understood as differential operators in the variables $(\xi, \eta)$. Furthermore, all norms are understood as norms of functions of $(\xi, \eta)$.

THEOREM 3.1. The Green's function $G(x, y ; \xi, \eta)$ from (3.3) satisfies

$$
|G(x, y ; \cdot)|_{1,1 ; \Omega} \leq C \varepsilon^{-1}
$$

Furthermore, for any ball $B(a, b ; \rho)$ of radius $\rho$ we have

$$
|G(x, y ; \cdot)|_{1,1 ; B(a, b ; \rho) \cap \Omega} \leq C \varepsilon^{-2} \rho,
$$

while for the ball $B(x, y ; \rho)$ centered at $(x, y)$ of radius $\rho$ we have

$$
|G(x, y ; \cdot)|_{2,1 ; \Omega \backslash B(x, y ; \rho)} \leq C \varepsilon^{-2} \ln (2+\varepsilon / \rho) .
$$

3.1. Constant-coefficient case. First, we shall establish a particular case of Theorem 3.1. Let $p:=\gamma^{2}$, where $\gamma=$ const $>0$, and let $\Omega$ be the quarter plane $\mathbb{R}_{+}^{2}=\{x, y>0\}$. In this particular case we denote the differential operator by $\bar{L}$ and the Green's function by $\bar{G}$, and for each $(x, y)$ we have

$$
\bar{L} \bar{G}(x, y ; \xi, \eta):=-\varepsilon^{2}\left[\bar{G}_{\xi \xi}+\bar{G}_{\eta \eta}\right]+\gamma^{2} \bar{G}=\delta(x-\xi) \delta(y-\eta), \quad \xi, \eta>0 .
$$

The fundamental solution for the differential operator $\bar{L}$ is

$$
g(x, y ; \xi, \eta):=\frac{1}{2 \pi \varepsilon^{2}} K_{0}\left(\frac{\gamma r}{\varepsilon}\right), \quad r:=\sqrt{(x-\xi)^{2}+(y-\eta)^{2}}
$$

Copyright (C) by SIAM. Unauthorized reproduction of this article is prohibited. 
where $K_{0}$ is the modified Bessel function of the second kind of order zero [1]. Hence the Green's function for this differential operator over the quarter plane is

$$
\bar{G}(x, y ; \xi, \eta)=g(x, y ; \xi, \eta)-g(-x, y ; \xi, \eta)-g(x,-y ; \xi, \eta)+g(-x,-y ; \xi, \eta) .
$$

Lemma 3.2. For $\bar{G}(x, y ; \xi, \eta)$ of (3.8), estimates (3.5) hold true, in which $G$ is replaced by $\bar{G}$ and $\Omega$ is replaced by $\mathbb{R}_{+}^{2}$.

Proof. We shall prove estimates (3.5) only for $\left\|\bar{G}_{\xi}\right\|_{1}$ and $\left\|\bar{G}_{\xi \xi}\right\|_{1}$ here, since the estimates for $\left\|\bar{G}_{\eta}\right\|_{1}$ and $\left\|\bar{G}_{\eta \eta}\right\|_{1}$ with $\left\|\bar{G}_{\xi \eta}\right\|_{1}$ are obtained similarly. Furthermore, it suffices to show (3.5) with $\bar{G}$ replaced by the first term $g(x, y ; \xi, \eta)$ of the representation (3.8) of $\bar{G}$, as the estimates for the other three terms are similar.

Since $K_{0}^{\prime}=-K_{1}$, where $K_{1}$ is the modified Bessel function of the second kind of order one, and $\partial r / \partial \xi=-(x-\xi) / r$, we get

$$
g_{\xi}(x, y ; \xi, \eta)=\frac{\gamma}{2 \pi \varepsilon^{3}} K_{1}\left(\frac{\gamma r}{\varepsilon}\right) \frac{x-\xi}{r} .
$$

Furthermore, a similar calculation invoking $K_{1}^{\prime}(s)=-K_{0}(s)-s^{-1} K_{1}(s)$ yields

$$
g_{\xi \xi}(x, y ; \xi, \eta)=\frac{\gamma^{2}}{2 \pi \varepsilon^{4}}\left[K_{0}\left(\frac{\gamma r}{\varepsilon}\right)+\frac{K_{1}(\gamma r / \varepsilon)}{\gamma r / \varepsilon}\right] \frac{(x-\xi)^{2}}{r^{2}}-\frac{\gamma}{2 \pi \varepsilon^{3}} K_{1}\left(\frac{\gamma r}{\varepsilon}\right) \frac{(y-\eta)^{2}}{r^{3}} .
$$

Hence we have

$$
\left|g_{\xi}\right| \leq C \varepsilon^{-3} K_{1}(s), \quad\left|g_{\xi \xi}(x, y ; \xi, \eta)\right| \leq C \varepsilon^{-4}\left[K_{0}(s)+\frac{K_{1}(s)}{s}\right], \quad s:=\frac{\gamma r}{\varepsilon} .
$$

To obtain the desired bounds for $\left\|g_{\xi}\right\|_{1}$ and $\left\|g_{\xi \xi}\right\|_{1}$, we represent these integral norms in polar coordinates and then substitute $r=\varepsilon \gamma^{-1} s$; note that $d \xi d \eta=r d r d \varphi=$ $C \varepsilon^{2} s d s d \varphi$. For $\left\|g_{\xi}\right\|_{1 ; \mathbb{R}^{2}}$, this yields

$$
\left\|g_{\xi}(x, y ; \cdot)\right\|_{1 ; \mathbb{R}^{2}} \leq C \varepsilon^{-3} \int_{\mathbb{R}^{2}} K_{1}(s) d \xi d \eta \leq C \varepsilon^{-1} \int_{0}^{\infty} s K_{1}(s) d s \leq C \varepsilon^{-1},
$$

where we also used $0<K_{1}(s)<C s^{-1} e^{-s}[1]$. Similarly,

$$
\left\|g_{\xi}(x, y ; \cdot)\right\|_{1 ; B(a, b ; \rho)} \leq C \varepsilon^{-3} \int_{B(x, y ; \rho)} K_{1}(s) d \xi d \eta \leq C \varepsilon^{-1} \int_{0}^{\gamma \rho / \varepsilon} s K_{1}(s) d s \leq C \varepsilon^{-2} \rho .
$$

Here replacing the integral over $B(a, b ; \rho)$ by the integral over $B(x, y ; \rho)$ yields an upper bound since $K_{1}$ is a positive decreasing function. Thus we obtained the desired estimates for $\left\|g_{\xi}\right\|_{1}$, which imply estimates (3.5a) and (3.5b) for $\left\|\bar{G}_{\xi}\right\|_{1}$.

Next, in a similar manner, we estimate $\left\|g_{\xi \xi}\right\|_{1}$ :

$$
\begin{aligned}
\left\|g_{\xi \xi}(x, y ; \cdot)\right\|_{1 ; \mathbb{R}^{2} \backslash B(x, y ; \rho)} & \leq C \varepsilon^{-2} \int_{\gamma \rho / \varepsilon}^{\infty} s\left[K_{0}(s)+\frac{K_{1}(s)}{s}\right] d s \leq C \varepsilon^{-2}\left[1+K_{0}\left(\frac{\rho}{\varepsilon}\right)\right] \\
& \leq C \varepsilon^{-2} \ln \left(2+\frac{\varepsilon}{\rho}\right),
\end{aligned}
$$

where, to get the second inequality, we used $0<K_{0}(s)<C s^{-1} e^{-s}$ and $K_{1}=-K_{0}^{\prime}$, while to get the final bound, we invoked $0<K_{0}(s)<C \ln \left(2+s^{-1}\right)$ [1]. This implies estimate (3.5c) for $\left\|\bar{G}_{\xi \xi}\right\|_{1}$.

Remark 3.1. An inspection of the proof of Lemma 3.2, in which we used the explicit representation (3.8), (3.7) of the Green's function in the constant-coefficient case, shows that the estimates of the Green's function in Theorem 3.1 are sharp. 
3.2. Smooth-coefficient case. Next, we shall use Lemma 3.2 to establish a variable-coefficient case of Theorem 3.1 under the additional assumption that

$$
\|p\|_{C^{0,1}(\bar{\Omega})} \leq C
$$

where $C^{0,1}(\bar{\Omega})$ is a standard Hölder space.

Lemma 3.3. Under condition (3.9), the Green's function $G(x, y ; \xi, \eta)$ from (3.3) satisfies estimates (3.5a) and (3.5c).

Proof. Fix $(x, y) \in \Omega$. Without loss of generality consider only the case of $x \leq 1 / 2$ and $y \leq 1 / 2$, as the other cases are similar. Let $\bar{G}$ be defined by (3.7), (3.8) with the frozen coefficient $\gamma^{2}:=p(x, y)$. By Lemma 3.2, estimates (3.5) hold true for $\bar{G}$. Hence is suffices to show that the function

$$
v=v(x, y ; \xi, \eta):=G(x, y ; \xi, \eta)-\omega(\xi, \eta) \bar{G}(x, y ; \xi, \eta),
$$

where $\omega(\xi, \eta)$ is a smooth cut-off function that equals 1 on $[0,3 / 4] \times[0,3 / 4]$ and vanishes on the boundaries $\xi=1$ and $\eta=1$, satisfies

$$
\varepsilon^{2}|v(x, y ; \cdot)|_{2,1 ; \Omega}+\varepsilon|v(x, y ; \cdot)|_{1,1 ; \Omega} \leq C .
$$

Clearly, $v=0$ for $(\xi, \eta) \in \partial \Omega$. Furthermore, using $L G=\bar{L} \bar{G}$ and (3.6), it is easy to check that $L v=\left(\gamma^{2}-p\right) \bar{G}+L[(\bar{G}-\omega \bar{G}]$, i.e.,

$$
\begin{gathered}
L v=-\varepsilon^{2}\left[v_{\xi \xi}+v_{\eta \eta}\right]+p(\xi, \eta) v=\phi \\
\phi=\phi_{1}+\phi_{2}, \quad \phi_{1}:=\left[\gamma^{2}-p(\xi, \eta)\right] \bar{G}, \quad \phi_{2}:=L[(1-\omega) \bar{G}] .
\end{gathered}
$$

Let the stretching transformation from $(\xi, \eta)$ to the new coordinates $\hat{\xi}:=(\xi-x) / \varepsilon$ and $\hat{\eta}:=(\eta-y) / \varepsilon$ map the original domain $\Omega$ into the domain $\hat{\Omega}$. Using the notation $\hat{w}(\hat{\xi}, \hat{\eta}):=w(x, y ; \xi, \eta)$ for any function $w$, rewrite $(3.12 \mathrm{a})$ as $-\triangle \hat{v}+\hat{p} \hat{v}=\hat{\phi}$. Combining this with $\hat{v}=0$ on $\partial \hat{\Omega}$ yields $\|\hat{v}\|_{2 ; \hat{\Omega}} \leq \beta^{-1 / 2}\|\hat{\phi}\|_{2 ; \hat{\Omega}}$ and, more importantly,

$$
\|\hat{v}\|_{2,2 ; \hat{\Omega}} \leq C_{1}\left(\|\hat{\phi}\|_{2 ; \hat{\Omega}}+\|\hat{v}\|_{2 ; \hat{\Omega}}\right) \leq C_{1}\left(1+\beta^{-1 / 2}\right)\|\hat{\phi}\|_{2 ; \hat{\Omega}}
$$

where the constant $C_{1}$ is independent of the size of the domain $\hat{\Omega}[17$, Chapter 3 , Lemma 8.1, p. 175]. Estimate (3.13), rewritten in the original variables $(\xi, \eta)$, implies that

$$
\varepsilon^{2}|v(x, y ; \cdot)|_{2,2 ; \Omega}+\varepsilon|v(x, y ; \cdot)|_{1,2 ; \Omega} \leq C\|\phi(x, y ; \cdot)\|_{2 ; \Omega} .
$$

Next, we claim that for $\phi$ from (3.12b) we have $\|\phi(x, y ; \cdot)\|_{2 ; \Omega} \leq C$. Indeed, $\phi_{2}=0$ for $(\xi, \eta) \in[0,3 / 4] \times[0,3 / 4]$ and $\left|\phi_{2}\right| \leq C$ outside. Furthermore, condition (3.9) implies that $\left|\gamma^{2}-p(\xi, \eta)\right| \leq C r$, where $\gamma^{2}=p(x, y)$. Hence $\left|\phi_{1}\right| \leq C \varepsilon^{-2} r K_{0}(\gamma r / \varepsilon)$, while

$$
\left\|\phi_{1}\right\|_{2}^{2} \leq C \varepsilon^{-2} \int_{\Omega}(r / \varepsilon)^{2} K_{0}^{2}(\gamma r / \varepsilon) d \xi d \eta \leq C \int_{0}^{\infty} s^{3} K_{0}^{2}(s) d s \leq C .
$$

Thus we have established that $\|\phi\|_{L_{2}(\Omega)} \leq C$. Combining this with (3.14) and $|v|_{k, 1} \leq$ $C|v|_{k, 2}$ for $k=1,2$, we obtain (3.11) and complete the proof. 
Note that condition (3.9), under which we now proved Theorem 3.1, is suitable for the particular linear case (3.1) of problem (1.1). However, if we consider the semilinear case (1.1) and linearize (2.10) to the form (3.1), then the coefficient $p$ depends on $u$ and $U$ and assumption (3.9) becomes unrealistic. Hence, we still have to prove Theorem 3.1 for the general case of $p$ satisfying only (3.2).

3.3. General case. Proof of Theorem 3.1. Let $\bar{G}$ be defined by (3.8), (3.7) with the coefficient $\gamma^{2}:=\beta$. Then, by the maximum/comparison principle, $L[\bar{G}-G]=$ $[p(\xi, \eta)-\beta] \bar{G} \geq 0$ combined with $\bar{G}-G \geq 0$ on $\partial \Omega$ implies that $0 \leq G \leq \bar{G}$.

Imitating the proof of Lemma 3.3, we arrive at (3.14), in which again $\left\|\phi_{2}\right\|_{2} \leq C$, while $\left|\gamma^{2}-p(\xi, \eta)\right| \leq C$ implies only that $\left|\phi_{1}\right| \leq C \varepsilon^{-2} K_{0}(\gamma r / \varepsilon)$. Hence $\left\|\phi_{1}\right\|_{2} \leq C \varepsilon^{-1}$ and $\|\phi\|_{2} \leq C \varepsilon^{-1}$. Combining this with (3.14) and $|v|_{k, 1 ; B(a, b ; \rho)} \leq C \rho|v|_{k, 2 ; \Omega}$ or $|v|_{k, 1 ; B(x, y ; \varepsilon)} \leq C \varepsilon|v|_{k, 2 ; \Omega}$ for $k=1,2$, we obtain

$$
\varepsilon|v(x, y ; \cdot)|_{1,1 ; B(a, b ; \rho)} \leq C \varepsilon^{-1} \rho
$$

and

$$
\varepsilon^{2}|v(x, y ; \cdot)|_{2,1 ; B(x, y ; \varepsilon)}+\varepsilon|v(x, y ; \cdot)|_{1,1 ; B(x, y ; \varepsilon)} \leq C ;
$$

compare with (3.11).

Combining (3.15) and (3.16) with Lemma 3.2, we observe that $G=v+\omega \bar{G}$ satisfies (3.5b), and furthermore, $G$ satisfies (3.5a) and (3.5c), with $\Omega$ replaced by $B(x, y ; \varepsilon)$. Hence, to complete the proof, it suffices to show that

$$
\varepsilon^{2}|G(x, y ; \cdot)|_{2,1 ; \Omega \backslash B(x, y ; \varepsilon)}+\varepsilon|G(x, y ; \cdot)|_{1,1 ; \Omega \backslash B(x, y ; \varepsilon)} \leq C .
$$

Next, divide the domain $\Omega \backslash B(x, y ; \varepsilon)$ into the subdomains $\mathcal{D}_{j}:=\{(\xi, \eta) \in \Omega$ : $\left.\rho_{j}<r<\rho_{j+1}\right\}$, where $j=0,1, \ldots$ and $\rho_{j}:=2^{j} \varepsilon$. Furthermore, $\mathcal{D}_{j} \subset \mathcal{D}_{j}^{\prime}:=$ $\mathcal{D}_{j-1} \cup \overline{\mathcal{D}}_{j} \cup \mathcal{D}_{j+1}$ so that $\operatorname{dist}\left(\partial \mathcal{D}_{j} \backslash \partial \Omega, \partial \mathcal{D}_{j}^{\prime} \backslash \partial \Omega\right) \geq \varepsilon / 2$.

Let the stretching transformation from $(\xi, \eta)$ to the new coordinates $\hat{\xi}:=(\xi-x) / \varepsilon$ and $\hat{\eta}:=(\eta-y) / \varepsilon$ map $\mathcal{D}_{j}$ into $\hat{\mathcal{D}}_{j}$. Imitating the proof of Lemma 3.3, we use the notation $\hat{G}(\hat{\xi}, \hat{\eta}):=G(x, y ; \xi, \eta)$ and rewrite the equation from (3.3) as $-\triangle \hat{G}+\hat{p} \hat{G}=0$ to get the local estimate

$$
\|\hat{G}\|_{2,2 ; \hat{\mathcal{D}}_{j}} \leq C_{2}\|\hat{G}\|_{2 ; \hat{\mathcal{D}}_{j}^{\prime}}
$$

[17, Chapter 3, estimate (8.6), p. 171]; compare with the global estimate (3.13). Here the constant $C_{2}$ is independent of $\varepsilon \operatorname{since} \operatorname{dist}\left(\partial \hat{\mathcal{D}}_{j} \backslash \partial \hat{\Omega}, \partial \hat{\mathcal{D}}_{j}^{\prime} \backslash \partial \hat{\Omega}\right) \geq 1 / 2$. Rewriting the above estimate in the variables $(\xi, \eta)$ yields

$$
\varepsilon^{2}|G(x, y ; \cdot)|_{2,2 ; \mathcal{D}_{j}}+\varepsilon|G(x, y ; \cdot)|_{1,2 ; \mathcal{D}_{j}} \leq C\|G(x, y ; \cdot)\|_{2, \mathcal{D}_{j}^{\prime}} \leq C\|g(x, y ; \cdot)\|_{2, \mathcal{D}_{j}^{\prime}} .
$$

Here we also used $|G| \leq \bar{G} \leq 4 g$; see (3.7), (3.8). Finally, since $|G|_{k, 1 ; \mathcal{D}_{j}} \leq C \rho_{j}|G|_{k, 2 ; \mathcal{D}_{j}}$ for $k=1,2$, we arrive at

$$
\varepsilon^{2}|G(x, y ; \cdot)|_{2,1 ; \mathcal{D}_{j}}+\varepsilon|G(x, y ; \cdot)|_{1,1 ; \mathcal{D}_{j}} \leq C \rho_{j}\|g(x, y ; \cdot)\|_{2, \mathcal{D}_{j}^{\prime}} .
$$

Note that

$$
\|g(x, y ; \cdot)\|_{2, \mathcal{D}_{j}^{\prime}}^{2} \leq C \varepsilon^{-2} \int_{\gamma \rho_{j-1} / \varepsilon}^{\gamma \rho_{j+1} / \varepsilon} s K_{0}^{2}(s) d s \leq\left. C \varepsilon^{-2} e^{-2 s}\right|_{\gamma \rho_{j+1} / \varepsilon} ^{\gamma \rho_{j-1} / \varepsilon} \leq C \varepsilon^{-2} e^{-\gamma \rho_{j} / \varepsilon}
$$

Copyright (c) by SIAM. Unauthorized reproduction of this article is prohibited. 
since for $r \geq \varepsilon / 2$ we have $g \leq C \varepsilon^{-2} s^{-1 / 2} e^{-s}$, where $s=\gamma r / \varepsilon$. Furthermore,

$$
\sum_{j=0}^{\infty} \rho_{j}\|g(x, y ; \cdot)\|_{2, \mathcal{D}_{j}^{\prime}} \leq C \sum_{j=0}^{\infty} \frac{\gamma\left(\rho_{j}-\rho_{j-1}\right)}{2 \varepsilon} e^{-\gamma \rho_{j} /(2 \varepsilon)} \leq C \int_{\gamma / 4}^{\infty} s e^{-s} d s \leq C
$$

since $\rho_{j}=2\left(\rho_{j}-\rho_{j-1}\right)$, while $e^{-s}$ is decreasing. Combining (3.20) with (3.19), we get (3.17) and thus complete the proof.

4. Stability properties of differential operators. The main result of the present section is the following stability theorem for the semilinear differential operator $T$ from (1.1), which we shall further apply to relation (2.10).

Consider the right-hand side $f$ in the special form

$$
f(x, y)=-\frac{\partial}{\partial x}\left[F_{1}(x, y)+\bar{F}_{1}(x, y)\right]-\frac{\partial}{\partial y}\left[F_{2}(x, y)+\bar{F}_{2}(x, y)\right]+\bar{f}(x, y),
$$

where $F_{1}, \bar{F}_{1}, F_{2}, \bar{F}_{2}, f \in L_{\infty}(\Omega)$ and

$$
\begin{array}{ll}
F_{1}(x, y)=A_{i}(y)\left(x-x_{i-1 / 2}\right) & \text { for }(x, y) \in\left(x_{i-1}, x_{i}\right) \times[0,1], \quad i=1, \ldots, N \\
F_{2}(x, y)=B_{j}(x)\left(y-y_{j-1 / 2}\right) & \text { for }(x, y) \in[0,1] \times\left(y_{j-1}, y_{j}\right), \quad j=1, \ldots, M
\end{array}
$$

while $x_{i-1 / 2}:=x_{i}-h_{i} / 2$ and $y_{j-1 / 2}:=y_{j}-k_{j} / 2$.

THEOREM 4.1. Let the function $b$ in (1.1) satisfy (1.2). Then, for any $v, w \in$ $W^{1,2}(\Omega)$ such that $T v(x, y)-T w(x, y)=f(x, y)$, where $f$ is defined by (4.1), and $v(x, y)=w(x, y)$ for $(x, y) \in \partial \Omega$, we have

$$
\begin{aligned}
\| v- & w\left\|_{\infty} \leq C \varepsilon^{-1}\left(\left\|\bar{F}_{1}\right\|_{\infty}+\left\|\bar{F}_{2}\right\|_{\infty}\right)+\beta^{-1}\right\| \bar{f} \|_{\infty} \\
& +C\left[\max _{i=1, \ldots, N}\left\{\frac{h_{i}^{2}}{\varepsilon^{2}} \max _{y \in[0,1]}\left|A_{i}(y)\right|\right\}+\max _{j=1, \ldots, M}\left\{\frac{k_{j}^{2}}{\varepsilon^{2}} \max _{x \in[0,1]}\left|B_{j}(x)\right|\right\}\right] \ln \left(2+\frac{\varepsilon}{\kappa}\right) .
\end{aligned}
$$

The proof is deferred to section 4.2

4.1. Linear reaction-diffusion. First, we address the linear problem (3.1), (3.2) with the right-hand side (4.1). Since the differential operator $L$ is linear, it is convenient to establish stability of $u$ with respect to various components of $f$ separately.

LEMma 4.2. There exists a unique solution $u \in L_{\infty}(\Omega)$ of problem (3.1), (3.2) with the right-hand side (4.1a). Furthermore, if $F_{1}=F_{2}:=0$, then

$$
\|u\|_{\infty} \leq \beta^{-1}\|\bar{f}\|_{\infty}+C \varepsilon^{-1}\left(\left\|\bar{F}_{1}\right\|_{\infty}+\left\|\bar{F}_{2}\right\|_{\infty}\right) .
$$

Proof. Since $L$ is linear, it suffices to establish the desired estimate in the following two cases. Case A: $f=\bar{f}$, while $\bar{F}_{1}=\bar{F}_{2}:=0$. Then estimate (4.2) is well known and follows from the maximum/comparison principle extended to functions in the Sobolev space $W^{1,2}[9$, section 8.1]. Case B: $\bar{f}:=0$. Now the desired estimate (4.2) follows from (3.4) combined with estimate (3.5a).

Lemma 4.3. Let $\bar{F}_{1}=\bar{F}_{2}=\bar{f}:=0$ in (4.1a). Then the solution $u \in L_{\infty}(\Omega)$ of problem (3.1), (3.2) with the right-hand side (4.1) satisfies

$\|u\|_{\infty} \leq C\left[\max _{i=1, \ldots, N}\left\{\frac{h_{i}^{2}}{\varepsilon^{2}} \max _{y \in[0,1]}\left|A_{i}(y)\right|\right\}+\max _{j=1, \ldots, M}\left\{\frac{k_{j}^{2}}{\varepsilon^{2}} \max _{x \in[0,1]}\left|B_{j}(x)\right|\right\}\right] \ln \left(2+\frac{\varepsilon}{\kappa}\right)$,

where $\kappa:=\min \left\{\min _{i}\left\{h_{i}\right\}, \min _{j}\left\{k_{j}\right\}\right\}$. 
Proof. It suffices to consider only the case of $f:=-\partial F_{1} / \partial x$, i.e., $F_{2}:=0$, as the case of $f:=-\partial F_{2} / \partial y$ is considered similarly, while our differential operator $L$ is linear.

Fix $(x, y)$ and denote $v(\xi, \eta):=G(x, y ; \xi, \eta)$. Then, by (3.4), we have

$$
u(x, y)=\int_{\Omega} F_{1}(\xi, \eta) v_{\xi}(\xi, \eta) d \xi d \eta=\sum_{i=1}^{N} \int_{\Omega_{i}} A_{i}(\eta)\left(\xi-x_{i-1 / 2}\right) v_{\xi}(\xi) d \xi d \eta
$$

where $\Omega_{i}:=\left(x_{i-1}, x_{i}\right) \times(0,1), i=1, \ldots, N$. Furthermore, let $x \in\left[x_{m-1 / 2}, x_{m+1 / 2}\right]$ for some $0<m<N$ (the cases of $x \in\left[0, x_{1 / 2}\right]$ and $x \in\left[x_{N-1 / 2}, 1\right]$ are similar) and introduce the rectangular domain

$$
\Omega^{\prime}:=\left(x_{m-1}, x_{m+1}\right) \times\left(y-\tilde{h}_{m}, y+\tilde{h}_{m}\right), \quad \text { where } \tilde{h}_{m}:=\min \left\{h_{m}, h_{m+1}\right\} / 2,
$$

so that

$$
B\left(x, y ; \tilde{h}_{m}\right) \subset \Omega^{\prime} \subset B\left(x_{m-1 / 2}, y ; h_{m}\right) \cup B\left(x_{m+1 / 2}, y ; h_{m+1}\right) .
$$

Clearly, (4.3) can be written as $u(x, y)=S_{1}+S_{2}$, where

$$
S_{1}=\sum_{i=1}^{N} \int_{\Omega_{i}} A_{i}(\eta)\left(\xi-x_{i-1 / 2}\right) \tilde{v}_{\xi}(\xi, \eta) d \xi d \eta,
$$

with $\tilde{v}:=v$ in $\Omega_{i} \backslash \Omega^{\prime}$ and $\tilde{v}:=0$ in $\Omega^{\prime}$, while

$$
S_{2}=\sum_{i=m}^{m+1} \int_{\Omega_{i} \cap \Omega^{\prime}} A_{i}(\eta)\left(\xi-x_{i-1 / 2}\right) v_{\xi}(\xi, \eta) d \xi d \eta .
$$

To estimate $S_{1}$, note that

$$
\tilde{v}_{\xi}(\xi, \eta)=\tilde{v}_{\xi}\left(x_{i-1}, \eta\right)+\int_{x_{i-1}}^{\xi} \tilde{v}_{\xi \xi}(s, \eta) d s \quad \text { for } \xi \in\left(x_{i-1}, x_{i}\right) .
$$

Here $\tilde{v}_{\xi \xi}$ is well defined, since the singularity of $v$ occurs at $(x, y)$, which is inside $\Omega^{\prime}$, and furthermore, for each $i$ and $\eta$ either $\tilde{v}_{\xi \xi}=v_{\xi \xi}$ or $\tilde{v}_{\xi \xi}=0$ for all $\xi \in\left(x_{i-1}, x_{i}\right)$. Combining (4.5) with $\int_{x_{i-1}}^{x_{i}}\left(\xi-x_{i-1 / 2}\right) d \xi=0$ yields

$$
\begin{aligned}
\left|\int_{x_{i-1}}^{x_{i}}\left(\xi-x_{i-1 / 2}\right) \tilde{v}_{\xi}(\xi, \eta) d \xi\right| & =\left|\int_{x_{i-1}}^{x_{i}} d \xi\left(\xi-x_{i-1 / 2}\right) \int_{x_{i-1}}^{\xi} \tilde{v}_{\xi \xi}(s, \eta) d s\right| \\
& \leq \frac{h_{i}^{2}}{4} \int_{x_{i-1}}^{x_{i}}\left|\tilde{v}_{\xi \xi}(s, \eta)\right| d s .
\end{aligned}
$$

Hence

$$
\begin{aligned}
\left|S_{1}\right| & \leq \sum_{i=1}^{N} \frac{h_{i}^{2}}{4} \int_{0}^{1}\left|A_{i}(\eta)\right| d \eta \int_{x_{i-1}}^{x_{i}}\left|\tilde{v}_{\xi \xi}(s, \eta)\right| d s \\
& \leq \max _{i=1, \ldots, N}\left\{\frac{h_{i}^{2}}{4} \max _{\eta \in[0,1]}\left|A_{i}(\eta)\right|\right\} \int_{\Omega}\left|\tilde{v}_{\xi \xi}(\xi, \eta)\right| d \xi d \eta .
\end{aligned}
$$

Copyright $@$ by SIAM. Unauthorized reproduction of this article is prohibited. 
Finally, recalling (4.4) and estimate (3.5c) for $v_{\xi \xi}=G_{\xi \xi}$, we get

$$
\int_{\Omega}\left|\tilde{v}_{\xi \xi}(\xi, \eta)\right| d \xi d \eta \leq \int_{\Omega \backslash B\left(x, y ; \tilde{h}_{m}\right)}\left|v_{\xi \xi}(\xi, \eta)\right| d \xi d \eta \leq C \varepsilon^{-2} \ln \left(2+\varepsilon / \tilde{h}_{m}\right)
$$

and thus the desired estimate for $S_{1}$ :

$$
\left|S_{1}\right| \leq C \max _{i=1, \ldots, N}\left\{\frac{h_{i}^{2}}{\varepsilon^{2}} \max _{\eta \in[0,1]}\left|A_{i}(\eta)\right|\right\} \ln \left(2+\frac{\varepsilon}{\kappa}\right) .
$$

It remains to obtain a similar estimate for $S_{2}$ :

$$
\begin{aligned}
S_{2} & \leq \sum_{i=m}^{m+1} h_{i} \max _{\eta \in[0,1]}\left|A_{i}(\eta)\right| \int_{B\left(x_{i-1 / 2}, y ; h_{i}\right)}\left|v_{\xi}(\xi, \eta)\right| d \xi d \eta \leq \sum_{i=m}^{m+1} \max _{\eta \in[0,1]}\left|A_{i}(\eta)\right| h_{i} \frac{C h_{i}}{\varepsilon^{2}} \\
& \leq C \max _{i}\left\{\frac{h_{i}^{2}}{\varepsilon^{2}} \max _{\eta \in[0,1]}\left|A_{i}(\eta)\right|\right\} .
\end{aligned}
$$

Here we used (4.4) and estimate (3.5b) for $v_{\xi}=G_{\xi}$.

4.2. Semilinear reaction-diffusion. Proof of Theorem 4.1. Using the standard linearization technique, we have $T v(x, y)-T w(x, y)=L[v(x, y)-w(x, y)]$, where the operator $L$ is linear and defined by (3.1) with the coefficient $p(x, y)=$ $\int_{0}^{1} b_{u}(x, y, w(x, y)+s[v(x, y)-w(x, y)]) d s$, which, by (1.2), satisfies condition (3.2). Hence the desired estimate follows from Lemmas 4.2 and 4.3 .

5. Analysis of the numerical method. Proof of Theorem 2.1. To complete the proof of Theorem 2.1, which we started in section 2, we shall invoke the following lemma.

LEMma 5.1. We have

$-\varepsilon^{2} \frac{\partial^{2} U^{\mathrm{I}}\left(x, y_{j}\right)}{\partial x^{2}}+q_{1}^{\mathrm{I}}\left(x, y_{j}\right)=\frac{\partial}{\partial x} F_{1}\left(x, y_{j}\right), \quad-\varepsilon^{2} \frac{\partial^{2} U^{\mathrm{J}}\left(x_{i}, y\right)}{\partial y^{2}}+q_{2}^{\mathrm{J}}\left(x_{i}, y\right)=\frac{\partial}{\partial y} F_{2}\left(x_{i}, y\right)$,

where

$$
F_{1}\left(x, y_{j}\right):=q_{1, i j}\left(x-x_{i-1 / 2}\right)+\frac{D_{x}^{-} q_{1, i j}}{2}\left(x_{i}-x\right)^{2}, \quad x \in\left(x_{i-1}, x_{i}\right),
$$

for $i=1, \ldots, N, j=0, \ldots, M$, and

$$
F_{2}\left(x_{i}, y\right):=q_{2, i j}\left(y-y_{j-1 / 2}\right)+\frac{D_{y}^{-} q_{2, i j}}{2}\left(y_{j}-y\right)^{2}, \quad y \in\left(y_{i-1}, y_{i}\right),
$$

for $i=0, \ldots, N, j=1, \ldots, M$.

Proof. We closely imitate the one-dimensional argument used in the proof of $[12$, Theorem 3.3] and give the proof here only for completeness.

It suffices to obtain the first desired relation, as the other one is similar. To simplify the presentation, within this proof, fix $y_{j}$ and use the notation $U^{\mathrm{I}}(x):=$ $U^{\mathrm{I}}\left(x, y_{j}\right), q_{1}^{\mathrm{I}}(x):=q_{1}^{\mathrm{I}}\left(x, y_{j}\right), q_{1, i}:=q_{1, i j}$, and $F_{1}(x):=F\left(x, y_{j}\right)$. Furthermore, for any function $v$, let $v^{\prime}:=\partial v / \partial x$. Thus we intend to show that $-\varepsilon^{2}\left(U^{\mathrm{I}}\right)^{\prime \prime}+q_{1}^{\mathrm{I}}=F_{1}^{\prime}$.

First, note that

$$
-\varepsilon^{2}\left(U^{\mathrm{I}}\right)^{\prime \prime}+q_{1}^{I}=-\left[\varepsilon^{2}\left(U^{\mathrm{I}}\right)^{\prime}+\int_{x}^{1} q_{1}^{I}(s) \mathrm{d} s\right]^{\prime} .
$$

Copyright (C) by SIAM. Unauthorized reproduction of this article is prohibited. 
Recalling (2.3) and (2.8), we observe that

$\varepsilon^{2}\left(U^{\mathrm{I}}\right)^{\prime}=\varepsilon^{2} D_{x}^{-} U_{i}^{\mathrm{I}}=\varepsilon^{2} D_{x}^{-} U_{N}^{\mathrm{I}}-\sum_{m=i}^{N-1} q_{1, m} \frac{h_{m}+h_{m+1}}{2}, \quad x \in\left(x_{i-1}, x_{i}\right), \quad i=1, \ldots, N$.

Now, substituting the above representation in (5.2) and omitting the derivative of the constant $\varepsilon^{2} D^{-} U_{N}^{\mathrm{I}}$, we arrive at $-\varepsilon^{2}\left(U^{\mathrm{I}}\right)^{\prime \prime}+q_{1}^{I}=\tilde{F}_{1}^{\prime}$, where

$$
\tilde{F}_{1}(x):=\sum_{m=i}^{N-1} q_{1, m} \frac{h_{m}+h_{m+1}}{2}-\int_{x}^{1} q_{1}^{I}(s) \mathrm{d} s, \quad x \in\left(x_{i-1}, x_{i}\right), \quad i=1, \ldots, N .
$$

A calculation shows that

$$
\sum_{m=i}^{N-1} q_{1, m} \frac{h_{m}+h_{m+1}}{2}=q_{1, i} \frac{h_{i}}{2}+\int_{x_{i}}^{1} q_{1}^{I}(s) \mathrm{d} s-q_{1, N} \frac{h_{N}}{2},
$$

and, omitting the derivative of another constant $q_{1, N} h_{N} / 2$, we obtain $\tilde{F}_{1}^{\prime}=F_{1}^{\prime}$, where

$$
F_{1}(x):=q_{1, i} \frac{h_{i}}{2}-\int_{x}^{x_{i}} q_{1}^{I}(s) \mathrm{d} s, \quad x \in\left(x_{i-1}, x_{i}\right), \quad i=1, \ldots, N .
$$

Thus we have obtained the desired relation $-\varepsilon^{2}\left(U^{\mathrm{I}}\right)^{\prime \prime}+q_{1}^{I}=F_{1}^{\prime}$, in which $F_{1}$ is defined by (5.3), and it remains to show that the definition of $F_{1}$ in (5.1a) is equivalent to (5.3). Indeed, by computing $\int_{x}^{x_{i}} q^{I}(s) \mathrm{d} s$ in (5.3) using $q_{1}^{I}(x)=q_{1, i}-\left(x_{i}-x\right) D^{-} q_{1, i}$, we get (5.1a).

Remark 5.1. One can easily check that $F_{1}$ and $F_{2}$ or (5.1) allow an alternative representation:

$$
\begin{array}{ll}
F_{1}\left(x, y_{j}\right):=q_{1, i-1, j}\left(x-x_{i-1 / 2}\right)+\frac{D_{x}^{-} q_{1, i j}}{2} O\left(h_{i}^{2}\right), & x \in\left(x_{i-1}, x_{i}\right), \\
F_{2}\left(x_{i}, y\right):=q_{2, i, j-1}\left(y-y_{j-1 / 2}\right)+\frac{D_{y}^{-} q_{2, i j}}{2} O\left(k_{j}^{2}\right), \quad y \in\left(y_{i-1}, y_{i}\right) .
\end{array}
$$

E.g., the new representation of $F_{1}$ follows from $q_{1, i j}=q_{1, i-1, j}+h_{i} D_{x}^{-} q_{1, i j}$.

Proof of Theorem 2.1 (continued from section 2). Extending $F_{1}$ and $F_{2}$ of (5.1) onto the whole domain $\bar{\Omega}$ by linear interpolation,

$$
F_{1}(x, y):=\left[F_{1}\left(x, y_{j}\right)\right]^{\mathrm{J}}, \quad F_{2}(x, y):=\left[F_{2}\left(x_{i}, y\right)\right]^{\mathrm{I}},
$$

we obtain (2.10). Now, invoking Theorem 4.1 yields

$$
\begin{aligned}
& \left\|U^{\mathrm{B}}-u\right\|_{\infty} \leq C\left[\max _{\substack{i=1, \ldots, N \\
j=0, \ldots, M}}\left\{\frac{h_{i}^{2}}{\varepsilon^{2}}\left|q_{1, i j}\right|\right\}+\max _{\substack{i=0, \ldots, N \\
j=1, \ldots, M}}\left\{\frac{k_{j}^{2}}{\varepsilon^{2}}\left|q_{2, i j}\right|\right\}\right] \ln \left(2+\frac{\varepsilon}{\kappa}\right) \\
& +C\left[\max _{\substack{i=1, \ldots, N \\
j=0, \ldots, M}}\left\{\frac{h_{i}^{2}}{\varepsilon}\left|D_{x}^{-} q_{1, i j}\right|\right\}+\max _{\substack{i=0, \ldots, N \\
j=1, \ldots, M}}\left\{\frac{k_{j}^{2}}{\varepsilon}\left|D_{y}^{-} q_{2, i j}\right|\right\}\right]+\beta^{-1}\left\|q-q^{\mathrm{B}}\right\|_{\infty}
\end{aligned}
$$

Combining this with (2.8) and the bilinear interpolation estimate

$$
\left\|q-q^{\mathrm{B}}\right\|_{\infty} \leq C\left[\max _{\substack{i=1, \ldots, N \\ j=0, \ldots, M}}\left\{h_{i}^{2}\left(1+\left|D_{x}^{-} U_{i j}\right|^{2}\right)\right\}+\max _{\substack{i=0, \ldots, N, j=1, \ldots, M}}\left\{k_{j}^{2}\left(1+\left|D_{y}^{-} U_{i j}\right|^{2}\right)\right\}\right],
$$

Copyright ( $)$ by SIAM. Unauthorized reproduction of this article is prohibited. 
we obtain a version of the desired a posteriori error estimate (2.7) in which the quantities $\min \left\{\left|D_{x}^{2} U_{i-1, j}\right|,\left|D_{x}^{2} U_{i j}\right|\right\}$ and $\min \left\{\left|D_{y}^{2} U_{i, j-1}\right|,\left|D_{y}^{2} U_{i j}\right|\right\}$ are replaced by $\left|D_{x}^{2} U_{i j}\right|$ and $\left|D_{y}^{2} U_{i j}\right|$, respectively.

By Remark 5.1, the quantities $\left|q_{1, i j}\right|$ and $\left|q_{2, i j}\right|$ in (5.4) can be replaced by $\min \left\{\left|q_{1, i-1, j}\right|,\left|q_{1, i j}\right|\right\}$ and $\min \left\{\left|q_{2, i, j-1}\right|,\left|q_{2, i j}\right|\right\}$, respectively. Combining this sharper version of (5.4) with (2.8) and (5.5) yields the desired estimate (2.7).

Finally, note that the interpolation error estimate (5.5), which we used, follows from $q-q^{B}=\left[q-q^{I}\right]+\left[q^{I}-\left(q^{I}\right)^{J}\right]$ combined with $\left|\partial^{2} q\left(x, y_{j}\right) / \partial x^{2}\right| \leq C\left(1+\left|D_{x}^{-} U_{i j}\right|^{2}\right)$ for $x \in\left(x_{i-1}, x_{i}\right)$ and $\left|\partial^{2} q\left(x_{i}, y\right) / \partial y^{2}\right| \leq C\left(1+\left|D_{y}^{-} U_{i j}\right|^{2}\right)$ for $y \in\left(y_{j-1}, y_{j}\right)$; see [3, Comment 2.15] for a similar argument.

6. Numerical results. The maximum norm a posteriori error estimate of Theorem 2.1 implies that

$$
\begin{gathered}
e:=\left\|U^{\mathrm{B}}-u\right\|_{\infty} \leq \tilde{C} \eta, \quad \eta:=\max \left\{\eta_{0}, \eta_{1}, \eta_{2}, \eta_{3}\right\}, \\
\eta_{l}:=\max \left\{\max _{\substack{i=1, \ldots, N \\
j=0, \ldots, M}}\left\{h_{i}^{2} M_{1, i j}^{(l)}\right\} ; \max _{\substack{i=0, \ldots, N \\
j=1, \ldots, M}}\left\{k_{j}^{2} M_{2, i j}^{(l)}\right\}\right\}, \quad l=0,1,2,3,
\end{gathered}
$$

where $\tilde{C}=C \ln (2+\varepsilon / \kappa)$, while $M_{1, i j}^{(0)}=M_{2, i j}^{(0)}=1$,

$$
\begin{aligned}
& M_{1, i j}^{(1)}:=\left|D_{x}^{-} U_{i j}\right|^{2}, \quad M_{1, i j}^{(2)}:=\min \left\{\left|D_{x}^{2} U_{i-1, j}\right|,\left|D_{x}^{2} U_{i j}\right|\right\}, \quad M_{1, i j}^{(3)}:=\varepsilon\left|D_{x}^{-} D_{x}^{2} U_{i j}\right|, \\
& M_{2, i j}^{(1)}:=\left|D_{y}^{-} U_{i j}\right|^{2}, \quad M_{2, i j}^{(2)}:=\min \left\{\left|D_{y}^{2} U_{i, j-1}\right|,\left|D_{y}^{2} U_{i j}\right|\right\}, \quad M_{2, i j}^{(3)}:=\varepsilon\left|D_{y}^{-} D_{y}^{2} U_{i j}\right| .
\end{aligned}
$$

Here $\eta_{l}$ and $M^{(l)}, l=1,2,3$, involve discrete analogues of $l$ th-order derivatives.

In this section we present numerical results on a priori chosen meshes to investigate the efficiency of the upper maximum norm error estimator $\eta$ in (6.1) and its components $\eta_{l}$. It is also of interest which of $\eta_{l}$ is the principal component in $\eta$ if any. We shall examine the errors $e$ and, more importantly, the quantities $\eta, e / \eta, \eta_{l}, e / \eta_{l}$ and their dependence on $\varepsilon, N=M$, and particular mesh choices.

We consider $\varepsilon=10^{-k}, k=1, \ldots, 10$, and two tensor-product meshes with $M=N$ : a variant of the layer-adapted mesh by Bakhvalov [4] and a simple uniform mesh; see Tables 6.1-6.5. Note that a Bakhvalov-type layer-adapted mesh was chosen for the numerical experiments, since it yields $\varepsilon$-uniform second-order accuracy [4, 13]. Furthermore, we expect a robust adaptive algorithm to generate a mesh that is very close to a Bakhvalov mesh, as in [14, section 6 and Figure 2].

To be precise, if $\varepsilon \leq \bar{\varepsilon}$, our Bakhvalov-type mesh is given by $x_{i}=y_{i}:=x(i / N)$ for $i=0,1, \ldots, N$, where $x(\xi):=\varepsilon \lambda \ln [b /(b-\xi)]$ for $\xi \in[0, \theta], x(1):=1$, and $x(\xi)$ is continuous on $[0,1]$ and linear on $[\theta, 1]$. We use the constants $b=1 / 2, \bar{\varepsilon}=b / \lambda$, and $\theta=b-\varepsilon \lambda$. The constant $\lambda$ will be specified later. For $\varepsilon>\bar{\varepsilon}$, the Bakhvalov mesh is defined to be a simple uniform mesh.

Our test problem is the linear problem (3.1), in which $p(x, y):=1$, and whose exact solution

$$
u(x, y):=\left(\cos (\pi x)-\frac{e^{-x / \varepsilon}-e^{-1 / \varepsilon}}{1-e^{-1 / \varepsilon}}\right)\left(1-y-\frac{e^{-y / \varepsilon}-e^{-1 / \varepsilon}}{1-e^{-1 / \varepsilon}}\right)
$$

exhibits boundary layers and a corner layer.

Copyright $@$ ㅇ by SIAM. Unauthorized reproduction of this article is prohibited. 
TABLE 6.1

Bakhvalov mesh, $\lambda=3$ : Maximum norm error e and the efficiency constant e/ $\eta$ for the upper maximum norm error estimator $\eta$.

\begin{tabular}{c|cc|cc|cc|cc}
\hline \multirow{2}{*}{$N$} & \multicolumn{2}{|c|}{$\varepsilon=10^{-1}$} & \multicolumn{2}{c|}{$\varepsilon=10^{-2}$} & \multicolumn{2}{c}{$\varepsilon=10^{-3}$} & \multicolumn{2}{c}{$\varepsilon=10^{-k}, k=4, \ldots, 10$} \\
\cline { 2 - 9 } & $e$ & $e / \eta$ & $e$ & $e / \eta$ & $e$ & $e / \eta$ & $e$ & $e / \eta$ \\
\hline 32 & $3.60 \mathrm{e}-3$ & $1.25 \mathrm{e}-1$ & $4.04 \mathrm{e}-3$ & $1.19 \mathrm{e}-1$ & $4.21 \mathrm{e}-3$ & $1.23 \mathrm{e}-1$ & $4.23 \mathrm{e}-3$ & $1.24 \mathrm{e}-1$ \\
64 & $9.16 \mathrm{e}-4$ & $1.25 \mathrm{e}-1$ & $1.02 \mathrm{e}-3$ & $1.19 \mathrm{e}-1$ & $1.07 \mathrm{e}-3$ & $1.24 \mathrm{e}-1$ & $1.08 \mathrm{e}-3$ & $1.24 \mathrm{e}-1$ \\
128 & $2.32 \mathrm{e}-4$ & $1.26 \mathrm{e}-1$ & $2.58 \mathrm{e}-4$ & $1.19 \mathrm{e}-1$ & $2.70 \mathrm{e}-4$ & $1.24 \mathrm{e}-1$ & $2.72 \mathrm{e}-4$ & $1.25 \mathrm{e}-1$ \\
256 & $5.85 \mathrm{e}-5$ & $1.27 \mathrm{e}-1$ & $6.46 \mathrm{e}-5$ & $1.19 \mathrm{e}-1$ & $6.78 \mathrm{e}-5$ & $1.24 \mathrm{e}-1$ & $6.82 \mathrm{e}-5$ & $1.25 \mathrm{e}-1$ \\
512 & $1.47 \mathrm{e}-5$ & $1.27 \mathrm{e}-1$ & $1.62 \mathrm{e}-5$ & $1.19 \mathrm{e}-1$ & $1.70 \mathrm{e}-5$ & $1.24 \mathrm{e}-1$ & $1.71 \mathrm{e}-5$ & $1.25 \mathrm{e}-1$ \\
\hline
\end{tabular}

TABLE 6.2

Bakhvalov mesh, $\lambda=3$ : Upper maximum norm error estimator $\eta$, its components $\eta_{1}, \eta_{2}, \eta_{3}$, and its efficiency constant $e / \eta$.

\begin{tabular}{c|cccc|cccc}
\hline \multirow{2}{*}{$N$} & \multicolumn{4}{|c|}{$\varepsilon=10^{-1}$} & \multicolumn{3}{c}{$\varepsilon=10^{-k}, k=4, \ldots, 10$} \\
\cline { 2 - 8 } & $\eta_{1}$ & $\eta_{2}$ & $\eta_{3}=\eta$ & $e / \eta$ & $\eta_{1}$ & $\eta_{2}$ & $\eta_{3}=\eta$ & $e / \eta$ \\
\hline 32 & $1.75 \mathrm{e}-2$ & $2.61 \mathrm{e}-2$ & $2.88 \mathrm{e}-2$ & $1.25 \mathrm{e}-1$ & $3.10 \mathrm{e}-2$ & $3.09 \mathrm{e}-2$ & $3.41 \mathrm{e}-2$ & $1.24 \mathrm{e}-1$ \\
64 & $4.72 \mathrm{e}-3$ & $6.96 \mathrm{e}-3$ & $7.31 \mathrm{e}-3$ & $1.25 \mathrm{e}-1$ & $8.26 \mathrm{e}-3$ & $8.25 \mathrm{e}-3$ & $8.65 \mathrm{e}-3$ & $1.24 \mathrm{e}-1$ \\
128 & $1.22 \mathrm{e}-3$ & $1.80 \mathrm{e}-3$ & $1.84 \mathrm{e}-3$ & $1.26 \mathrm{e}-1$ & $2.13 \mathrm{e}-3$ & $2.13 \mathrm{e}-3$ & $2.18 \mathrm{e}-3$ & $1.25 \mathrm{e}-1$ \\
256 & $3.11 \mathrm{e}-4$ & $4.57 \mathrm{e}-4$ & $4.62 \mathrm{e}-4$ & $1.27 \mathrm{e}-1$ & $5.41 \mathrm{e}-4$ & $5.41 \mathrm{e}-4$ & $5.47 \mathrm{e}-4$ & $1.25 \mathrm{e}-1$ \\
512 & $7.86 \mathrm{e}-5$ & $1.15 \mathrm{e}-4$ & $1.16 \mathrm{e}-4$ & $1.27 \mathrm{e}-1$ & $1.36 \mathrm{e}-4$ & $1.36 \mathrm{e}-4$ & $1.37 \mathrm{e}-4$ & $1.25 \mathrm{e}-1$ \\
\hline
\end{tabular}

TABLE 6.3

Uniform mesh: Maximum norm error e and the efficiency constant $e / \eta_{2}$ for the component $\eta_{2}$ of the upper maximum norm error estimator $\eta$.

\begin{tabular}{c|cc|cc|cc|cc}
\hline \multirow{2}{*}{$N$} & \multicolumn{2}{|c|}{$\varepsilon=10^{-2}$} & \multicolumn{2}{c|}{$\varepsilon=10^{-3}$} & \multicolumn{2}{c}{$\varepsilon=10^{-4}$} & \multicolumn{2}{c}{$\varepsilon=10^{-k}, k=5, \ldots, 10$} \\
\cline { 2 - 9 } & $e$ & $e / \eta_{2}$ & $e$ & $e / \eta_{2}$ & $e$ & $e / \eta_{2}$ & $e$ & $e / \eta_{2}$ \\
\hline 32 & $3.45 \mathrm{e}-1$ & $4.17 \mathrm{e}-1$ & $5.68 \mathrm{e}-1$ & $5.71 \mathrm{e}-1$ & $5.77 \mathrm{e}-1$ & $5.78 \mathrm{e}-1$ & $5.78 \mathrm{e}-1$ & $5.79 \mathrm{e}-1$ \\
64 & $1.60 \mathrm{e}-1$ & $2.77 \mathrm{e}-1$ & $5.54 \mathrm{e}-1$ & $5.59 \mathrm{e}-1$ & $5.77 \mathrm{e}-1$ & $5.77 \mathrm{e}-1$ & $5.78 \mathrm{e}-1$ & $5.78 \mathrm{e}-1$ \\
128 & $5.40 \mathrm{e}-2$ & $1.91 \mathrm{e}-1$ & $5.11 \mathrm{e}-1$ & $5.28 \mathrm{e}-1$ & $5.76 \mathrm{e}-1$ & $5.77 \mathrm{e}-1$ & $5.78 \mathrm{e}-1$ & $5.78 \mathrm{e}-1$ \\
256 & $1.57 \mathrm{e}-2$ & $1.53 \mathrm{e}-1$ & $4.04 \mathrm{e}-1$ & $4.56 \mathrm{e}-1$ & $5.73 \mathrm{e}-1$ & $5.74 \mathrm{e}-1$ & $5.78 \mathrm{e}-1$ & $5.78 \mathrm{e}-1$ \\
512 & $4.21 \mathrm{e}-3$ & $1.35 \mathrm{e}-1$ & $2.20 \mathrm{e}-1$ & $3.24 \mathrm{e}-1$ & $5.63 \mathrm{e}-1$ & $5.66 \mathrm{e}-1$ & $5.78 \mathrm{e}-1$ & $5.78 \mathrm{e}-1$ \\
\hline
\end{tabular}

TABLE 6.4

Uniform mesh: The components $\eta_{2}$ and $\eta_{3}$ of the upper maximum norm error estimator $\eta$ and the efficiency constant $e / \eta_{2}$ for $\eta_{2}$.

\begin{tabular}{c|rcc|rrc|rcc}
\hline & \multicolumn{3}{|c|}{$\varepsilon=10^{-4}$} & \multicolumn{3}{c|}{$\varepsilon=10^{-7}$} & \multicolumn{3}{c}{$\varepsilon=10^{-10}$} \\
\cline { 2 - 10 }$N$ & \multicolumn{1}{|c}{$\eta_{2}$} & $\eta_{3}=\eta$ & $e / \eta_{2}$ & \multicolumn{1}{c}{$\eta_{2}$} & \multicolumn{1}{c}{$\eta_{3}=\eta$} & $e / \eta_{2}$ & $\eta_{2}$ & $\eta_{3}=\eta$ & $e / \eta_{2}$ \\
\hline 32 & $9.99 \mathrm{e}-1$ & $3.12 \mathrm{e}+2$ & $5.78 \mathrm{e}-1$ & $9.99 \mathrm{e}-1$ & $3.12 \mathrm{e}+5$ & $5.79 \mathrm{e}-1$ & $9.99 \mathrm{e}-1$ & $3.12 \mathrm{e}+8$ & $5.79 \mathrm{e}-1$ \\
64 & $1.00 \mathrm{e}+0$ & $1.56 \mathrm{e}+2$ & $5.77 \mathrm{e}-1$ & $1.00 \mathrm{e}+0$ & $1.56 \mathrm{e}+5$ & $5.78 \mathrm{e}-1$ & $1.00 \mathrm{e}+0$ & $1.56 \mathrm{e}+8$ & $5.78 \mathrm{e}-1$ \\
128 & $9.99 \mathrm{e}-1$ & $7.81 \mathrm{e}+1$ & $5.77 \mathrm{e}-1$ & $1.00 \mathrm{e}+0$ & $7.81 \mathrm{e}+4$ & $5.78 \mathrm{e}-1$ & $1.00 \mathrm{e}+0$ & $7.81 \mathrm{e}+7$ & $5.78 \mathrm{e}-1$ \\
256 & $9.99 \mathrm{e}-1$ & $3.90 \mathrm{e}+1$ & $5.74 \mathrm{e}-1$ & $1.00 \mathrm{e}+0$ & $3.91 \mathrm{e}+4$ & $5.78 \mathrm{e}-1$ & $1.00 \mathrm{e}+0$ & $3.91 \mathrm{e}+7$ & $5.78 \mathrm{e}-1$ \\
512 & $9.95 \mathrm{e}-1$ & $1.95 \mathrm{e}+1$ & $5.66 \mathrm{e}-1$ & $1.00 \mathrm{e}+0$ & $1.95 \mathrm{e}+4$ & $5.78 \mathrm{e}-1$ & $1.00 \mathrm{e}+0$ & $1.95 \mathrm{e}+7$ & $5.78 \mathrm{e}-1$ \\
\hline
\end{tabular}

TABLE 6.5

Bakhvalov mesh, $\lambda=1$ : Maximum norm error e, upper maximum norm error estimator $\eta$, its components $\eta_{1}, \eta_{2}, \eta_{3}$, and its efficiency constant $e / \eta$.

\begin{tabular}{c|ccccc|ccccc}
\hline \multirow{3}{*}{$N$} & \multicolumn{3}{|c|}{$\varepsilon=10^{-5}$} & \multicolumn{4}{c}{$\varepsilon=10^{-10}$} \\
\cline { 2 - 11 } & $e$ & $\eta_{1}$ & $\eta_{2}$ & $\eta_{3}=\eta$ & $e / \eta$ & $e$ & $\eta_{1}$ & $\eta_{2}$ & $\eta_{3}=\eta$ & $e / \eta$ \\
\hline 32 & $7.50 \mathrm{e}-2$ & $9.60 \mathrm{e}-3$ & $1.67 \mathrm{e}-2$ & $2.13 \mathrm{e}-1$ & $3.52 \mathrm{e}-1$ & $9.48 \mathrm{e}-2$ & $9.61 \mathrm{e}-3$ & $1.58 \mathrm{e}-2$ & $2.41 \mathrm{e}-1$ & $3.93 \mathrm{e}-1$ \\
64 & $3.81 \mathrm{e}-2$ & $2.41 \mathrm{e}-3$ & $8.37 \mathrm{e}-3$ & $1.05 \mathrm{e}-1$ & $3.64 \mathrm{e}-1$ & $4.91 \mathrm{e}-2$ & $2.41 \mathrm{e}-3$ & $7.93 \mathrm{e}-3$ & $1.20 \mathrm{e}-1$ & $4.09 \mathrm{e}-1$ \\
128 & $1.89 \mathrm{e}-2$ & $6.02 \mathrm{e}-4$ & $4.22 \mathrm{e}-3$ & $5.15 \mathrm{e}-2$ & $3.66 \mathrm{e}-1$ & $2.49 \mathrm{e}-2$ & $6.02 \mathrm{e}-4$ & $3.98 \mathrm{e}-3$ & $5.94 \mathrm{e}-2$ & $4.19 \mathrm{e}-1$ \\
256 & $9.17 \mathrm{e}-3$ & $1.51 \mathrm{e}-4$ & $2.13 \mathrm{e}-3$ & $2.52 \mathrm{e}-2$ & $3.64 \mathrm{e}-1$ & $1.25 \mathrm{e}-2$ & $1.51 \mathrm{e}-4$ & $1.99 \mathrm{e}-3$ & $2.95 \mathrm{e}-2$ & $4.23 \mathrm{e}-1$ \\
512 & $4.40 \mathrm{e}-3$ & $3.76 \mathrm{e}-5$ & $1.07 \mathrm{e}-3$ & $1.23 \mathrm{e}-2$ & $3.58 \mathrm{e}-1$ & $6.22 \mathrm{e}-3$ & $3.76 \mathrm{e}-5$ & $9.96 \mathrm{e}-4$ & $1.46 \mathrm{e}-2$ & $4.25 \mathrm{e}-1$ \\
\hline
\end{tabular}

Copyright $@$ ㅇ by SIAM. Unauthorized reproduction of this article is prohibited. 
Tables 6.1 and 6.2 present numerical results for the Bakhvalov mesh with $\lambda=3$. This mesh yields $\varepsilon$-uniform second-order accuracy in the maximum norm; i.e., ultimately, we would like to be able to construct a similar adaptive mesh. We observe agreement with our theoretical estimate (6.1). Not only does $e / \eta$ stabilizesee Table 6.1 -but it becomes very close to the linear interpolation error constant $1 / 8=1.25 e-1$. Table 6.2 is given to compare the components $\eta_{l}$ of $\eta$. We observe that $\eta_{2} \approx \eta_{3}=\eta$. Furthermore, for $\varepsilon \leq 10^{-2}$ we have $\eta_{1} \approx \eta_{2} \approx \eta_{3}$, while for $\varepsilon=10^{-1}$ the quantity $\eta_{1}$ is dominated by $\eta_{2}$ and $\eta_{3}$. The quantity $\eta_{0}$ is not presented, since it is negligible and, furthermore, known a priori.

When uniform meshes are used - see Tables 6.3 and 6.4 - the boundary layers are not resolved and $e=O(1)$. This is indicated by $\eta=\eta_{3}$ blowing up even more significantly than $e$. Unlike $\eta_{3}$ the component $\eta_{2}$ remains bounded. Thus both $\eta_{2}$ and $\eta_{3}$ not being small correctly indicates that the method is inaccurate. But $\eta_{2}$ better reflects the actual errors since $e / \eta_{2} \approx$ const $=0.58$ in Table 6.4 .

Finally we consider the Bakhvalov mesh with $\lambda=1$; see Table 6.5. Since the condition $\lambda>2$, which implies $\varepsilon$-uniform second-order accuracy for our test problem $[4,13]$, is violated, the errors slightly decrease as $\varepsilon \rightarrow 0$. We observe that $\eta_{1}$ is too small compared to $\eta$ and $e$.

In summary, for our test problem on the meshes considered, the error estimator $\eta$ indicates correctly whether or not the method is $\varepsilon$-uniformly accurate. Furthermore, we observe that the quantity $\eta=\eta_{3}$ might blow up (see Table 6.4), while the component $\eta_{1}$ is sometimes too optimistic (see Table 6.5). The component $\eta_{2}$ seems the most relevant estimator for the actual error $e$. Besides, $\eta_{2}$ does not blow up, like $\eta_{3}$, and hence seems a suitable error indicator for a posteriori mesh construction.

\section{REFERENCES}

[1] M. Abramowitz And I. A. Stegun, Handbook of Mathematical Functions with Formulas, Graphs, and Mathematical Tables, National Bureau of Standards Applied Mathematics Series 55, U.S. Government Printing Office, Washington, D.C., 1964.

[2] M. AinswORTH AND I. BABUŠKA, Reliable and robust a posteriori error estimation for singularly perturbed reaction-diffusion problems, SIAM J. Numer. Anal., 36 (1999), pp. 331-353.

[3] T. Apel, Anisotropic Finite Elements: Local Estimates and Applications, Teubner, Stuttgart, 1999.

[4] N. S. BAKhVAlov, Towards optimization of methods for solving boundary value problems in the presence of a boundary layer, Zh. Vychisl. Mat. i Mat. Fiz., 9 (1969), pp. 841-859 (in Russian).

[5] I. BoglaEv, On monotone iterative methods for a nonlinear singularly perturbed reactiondiffusion problem, J. Comput. Appl. Math., 162 (2004), pp. 445-466.

[6] L. Chen, P. Sun, And J. Xu, Optimal anisotropic simplicial meshes for minimizing interpolation errors in $L_{p}$-norm, Math. Comp., 76 (2007), pp. 179-204.

[7] C. Clavero, J. L. Gracia, and E. O'Riordan, A parameter robust numerical method for a two dimensional reaction-diffusion problem, Math. Comp., 74 (2005), pp. 1743-1758.

[8] E. F. D'Azevedo, Optimal triangular mesh generation by coordinate transformation, SIAM J. Sci. Statist. Comput., 12 (1991), pp. 755-786.

[9] D. Gilbarg and N. S. Trudinger, Elliptic Partial Differential Equations of Second Order, Springer-Verlag, Berlin, 1998.

[10] W. Huang, Variational mesh adaptation: Isotropy and equidistribution, J. Comput. Phys., 174 (2001), pp. 903-924.

[11] N. Kopteva, Maximum norm a posteriori error estimates for a one-dimensional convectiondiffusion problem, SIAM J. Numer. Anal., 39 (2001), pp. 423-441.

[12] N. Kopteva, Maximum norm a posteriori error estimates for a $1 D$ singularly perturbed semilinear reaction-diffusion problem, IMA J. Numer. Anal., 27 (2007), pp. 576-592.

[13] N. Kopteva, Maximum norm error analysis of a $2 d$ singularly perturbed semilinear reactiondiffusion problem, Math. Comp., 76 (2007), pp. 631-646. 
[14] N. Kopteva And M. Stynes, A robust adaptive method for a quasi-linear one-dimensional convection-diffusion problem, SIAM J. Numer. Anal., 39 (2001), pp. 1446-1467.

[15] G. KUNERT AND R. VERFÜRTH, Edge residuals dominate a posteriori error estimates for linear finite element methods on anisotropic triangular and tetrahedral meshes, Numer. Math., 86 (2000), pp. 283-303.

[16] G. KUNERT, Robust a posteriori error estimation for a singularly perturbed reaction-diffusion equation on anisotropic tetrahedral meshes. A posteriori error estimation and adaptive computational methods, Adv. Comput. Math., 15 (2001), pp. 237-259.

[17] O. A. Ladyzhenskaya and N. N. URAL'tseva, Linear and Quasilinear Elliptic Equations, Academic Press, New York, 1968.

[18] T. LINß, Maximum-norm error analysis of a non-monotone FEM for a singularly perturbed reaction-diffusion problem, BIT, 47 (2007), pp. 379-391.

[19] T. Linß And M. STYNeS, The SDFEM on Shishkin meshes for linear convection-diffusion problems, Numer. Math., 87 (2001), pp. 457-484.

[20] J. J. H. Miller, E. O'Riordan, And G. I. Shishrin, Solution of Singularly Perturbed Problems with $\varepsilon$-uniform Numerical Methods-Introduction to the Theory of Linear Problems in One and Two Dimensions, World Scientific, Singapore, 1996.

[21] R. H. Nochetto, Pointwise a posteriori error estimates for elliptic problems on highly graded meshes, Math. Comp., 64 (1995), pp. 1-22.

[22] A. H. Schatz AND L. B. WAhlBin, On the finite element method for singularly perturbed reaction-diffusion problems in two and one dimensions, Math. Comp., 40 (1983), pp. $47-88$.

Copyright (c) by SIAM. Unauthorized reproduction of this article is prohibited. 